\title{
Notes on transliteration and spelling
}

In transliterating from Georgian and Russian into English we have generally used a modified Library of Congress Transliteration System. However, familiar names that are customarily used in English, for example Chavchavadze, Bariatishvili, or Alexander and Nicholas remain in their anglicized form (unless they appear as part of a Georgian or Russian title in the footnotes). In the chapter by Jeremy Smith, the Georgian names were retained in their Russian spelling, as all the sources were in Russian. 
